\title{
Advertising and Provision of Residential Infrastructure ${ }^{\dagger}$
}

\author{
Tatsuaki KURODA*
}

\section{Introduction}

Butters [2] has established the existence of equilibrium price distributions for a market where all buyers are identical and a single homogeneous good (i.e., there is no horizontal or vertical differentiation) is being traded. Balcer [1] extended Butters' model to incorporate location: sellers and buyers are spread uniformly on a surface ${ }^{1}$. Balcer's model can be interpreted in the context of markets for differentiated products which could be manufactured at the same unit cost (i.e., there is horizontal differentiation). In practice, however, many products are differentiated vertically in quality which is proportionate to the cost of production. In the case of land markets, a typical example is the provision level of residential infrastructure which is supplied by land developers. Moreover, if households are homogeneous and information is perfect, the provision level of infrastructure would not vary. This is a very special characteristic of infrastructure, as it is attached to land which a household usually purchases or rents just one block each ${ }^{2}$.

The purpose of this paper is to examine the role of information in the provision of infrastructure as well as in the diversity of the land rents in a land market. To do so, we focus on a specific land market in which land developers advertise the rent and the provision level of infrastructure of the land and homogeneous households have no alternative sources of information. We adopt a structure of information transmission following Butters' model which assumes a random process in the allocation of advertisements from the sellers. Thus, one might say that the substantial goal of this paper is to extend the basic part of Butters' model to (two-dimensional) joint distributions (i.e., rent and provision level of infrastructure). In this case, however, it should be noted that the good traded in the market (i.e., lots of land) may be heterogeneous (i.e., differentiated) in the level of attached infrastructure.

* Kyoto Institute of Economic Research, Kyoto University

$\dagger$ The author wishes to thank Haruo Imai, Makoto Tawada, Yasuhiko Ohishi, Fumiko Seo, Tony Smith, Masahisa Fujita, Ronald Miller, Dong-Ju Kim, and two anonymous referees for their helpful comments.

1 See Grossman and Shapiro [4] for another model of differentiated products, which employs an information technology similar to that in Butters' model.

2 If land was a good such that a household may consume several kinds at the same time, the welfare level of households would be better off as the provision level of infrastructure varies. Hence, in this case, even though information is perfect and households are homogeneous, the provision level of infrastructure would vary. This effect was first emphasized by Stahl [6]. Fujita [3] calls it "complementarity effect." 
Based on our model, we obtained the main results as follows. (1) In equilibrium, all combinations of rent and provision levels at which they are advertised are distributed on a curve which coincides with the (imaginary) income expansion path of households ; (2) The equilibrium number of advertising messages is socially optimal.

Our results are consistent with Balcer's observation that "if consumers are all alike, there is diversity in the behavior of producers". Furthermore, our results imply that the producers' choices happen to also be optimal for consumers, given the structure of information transmission.

The organization of this paper is as follows. In Section 2, the model is developed. Section 3 discusses results of the model from several points of view. Finally, a brief conclusion is presented in Section 4.

\section{The Model}

\subsection{Assumptions of the Model}

(A.1) There are many land developers and households in a land market.

(A.2) All households are homogeneous, i.e., they have the same income $Y$ and the same utility function $U(z, k)$ where $z$ represents the amount of the composite good, and $k$ the average provision level of residential infrastructure (per household) which is attached to the lot where the household resides ${ }^{3}$. The composite good is chosen as the numeraire.

(A.3) The utility function of households is defined for each positive combination of $z$ and $k$, and is continuous, strictly quasi-concave and strictly increasing in each argument. The indifference curves are smooth and do not intercept at the axes.

(A.4) The developers rent the raw land from the absentee (original) landlords at an agricultural rent $R_{A}$ (per lot) which is a constant, and provide infrastructure at some level $k$ (per lot) which each developer may choose.

(A.5) All the rental land is the similar, regardless of developer, except for the rent $R$ and the provision level of infrastructure $k$ (thus, the lot size does not vary).

(A.6) Land developers can distribute advertising messages informing households of the rent and provision level of infrastructure. In addition, it is assumed that the contents of advertisements are true (i.e., developers do not lie) $)^{4}$.

(A.7) We consider only a representative period. A new set of households enters the land

3 " $\mathrm{k}$ " in interpreted as the composite infrastructure. We implicitly assume that the infrastructure supplied by developers [e.g., (narrow) street, sewerage, water supply, (small) park, etc.] is very congestible and thus resembles a private good. Moreover, its scale economy is negligible. In addition, we omit discussion about local public goods which are (relatively) uncongestible or have large scale economies [e.g., main lines, libraries, large parks, fire stations, etc.] which are supposed to be provided uniformly by the local governments. Hence, in this paper, the provision level of residential infrastructure is evaluated approximately at the average (per hosuehold). Furthermore, " $k$ " can be interpreted as the (total) housing investment per lot, assuming that any husesholds cannot share a single lot.

4 Alternatively, according to the distinction by Nelson [5] we may assume that the provision level of infrastructure considered is not the "experience quality" but the "search quality" with no search cost. 
market. They may receive the advertisements. At the end of the period, they must rent exactly one lot or forfeit their opportunity to rent ${ }^{5}$.

(A.8) A parameter $b$ represents the cost to each developer of reaching a single household with a single advertisement. In other words, a single advertisement costs $b$ and will reach a single household (i.e., there are no economies of scale in advertising).

(A.9) Advertisements are distributed randomly among households, with each household having an equal chance of receiving each message. Moreover, the assignment of each advertisement to a household is independent of the assignments of any other advertisements, including those sent out by the same developer.

(A.10) Households receive messages free of charge and cannot influence the probability of receiving them. They have no other way of receiving information about the land (they cannot search for land). Thus, if they receive no messages, they cannot rent a lot of land. Those households who would have rented, but cannot because they do not receive a message, may be termed "potential households". Those who receive messages and rent a lot of land may be called "actual households". The term "household" will be used to include both categories.

(A.11) At the end, each actual household rents from the developer whose lot it most favors. If there are several such developers, the household chooses any one with equal probability.

(A.12) Each developer may change the area of his land (i.e., the number of lots) freely corresponding to demand, that is, his inventory costs are zero.

(A.13) All developers know the incomes of households, as well as the utility function, and the joint distribution of rents and provision levels of infrastructure advertised by other developers. They do not know which messages, if any, are received by any specific household.

(A.14) Each developer chooses the rent and the provision level of infrastructure so as to maximize his profits, given the behavior of other developers.

\subsection{Distribution of Rent and Provision Level of Infrastructure}

Since the source of information about the land is limited to the advertising messages received by households and the budget constraint of households is given by $z+R=Y$, the behavior of households is expressed as follows :

$$
\max _{(R, k) \varepsilon \Omega} U(Y-R, k),
$$

where $\Omega$ is the set of information presented in advertising messages received by each household. See fig. 1 for an illustration. In this figure, a household who receives three advertisements $a_{1}, a_{2}$, and $a_{3}$ will choose $a_{1}$ because it is the most favored combination of $R$ and $k$ for the household.

5 Alternatively, we might assume that $\left.\lim _{z \rightarrow 0} U(z, k)\right|_{k>0}=\left.\lim _{k \rightarrow 0} U(z, k)\right|_{z>0}=\lim _{\substack{k \rightarrow 0 \\ z \rightarrow 0}} U(z, k)=\underline{u}$, where $\underline{u}$ is the utility level when a household does not rent a lot by the end of the period. 


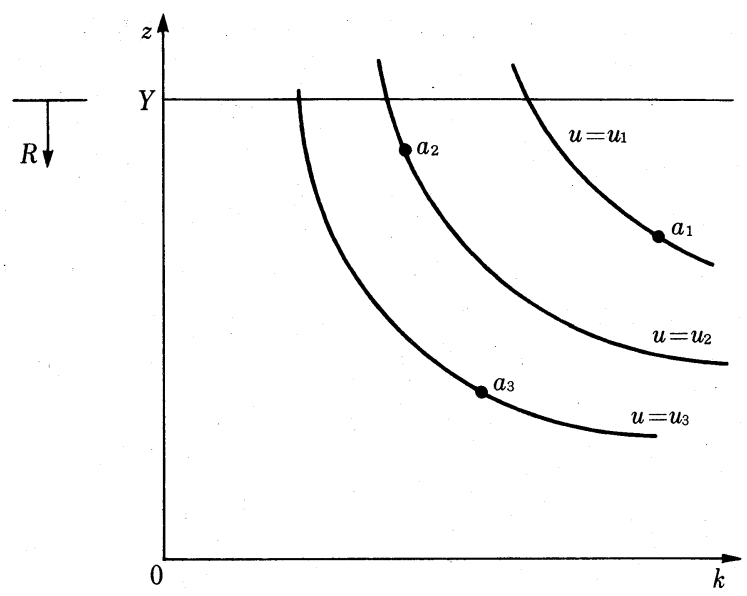

Fig. 1 The preference of households

Our major question is how the contents of advertisements are distributed in the twodimensional space of $(R, k)$. Since developers would not send an advertisement in which $R \geq Y$ by (A.3) and (A.13), we focus on the $(R, k)$ [or $(z, k)]$ space as depicted in fig. 1 .

To analyze the distribution, we need additional definitions as follows. Let $D$ and $H$ be the number of developers and households, respectively. Denoted by $M(R, k)$, the number of advertising messages sent out which are more favored than or indifferent to the lot of $(R, k)$ by households, divided by the number of households $H$. Thus, $M(Y, 0)$ [ = $M(R, 0)=M(Y, k)$ for $\forall R, \forall k$, respectively] equals the ratio of total advertisements to the number of households. Let $m(R, k)$ be the number of messages sent out which are indifferent to the lot of $(R, k)$ divided by $H$. Let $\mu(R, k)$ be the number of messages sent out at $(R, k)$ divided by $H$. Likewise, $C(R, k)$ denotes the number of rental contracts at the combinations of rents and provision levels of infrastructure which are more favored than or indifferent to the lot of $(R, k)$ for households, divided by the number of households $H$. Since we adopted the information structure of Butters, $C(Y, 0)[=C(R, 0)=C(Y, k)$ for $\forall R, \forall k$, respectively] $<1$. Let $c(R, k)$ be the number of contracts at the combinations of rents and provision levels of infrastructure which are indifferent to the lot of $(R, k)$ divided by $H$. Let $\gamma(R, k)$ be the number of contracts at $(R, k)$ divided by $H$.

For the same reason as in Butters' model, our main results can be obtained only in the limit where $D$ and $H$ approach infinity. ${ }^{6}$ Asterisks $\left({ }^{*}\right)$ mark the limits of some functions defined in this paper (e.g., $M^{*}, C^{*}$, etc.). Moreover, we limit our discussion to cases in which the strategies of developers are pure, and thus we adopt the concept of a Nash $\varepsilon$ equilibrium if the case is not the limit.

Next, let us consider the behavior of land developers. As assumed in (A.14), developers try to maximize profits by choosing a combination of rent and provision level of infrastructure which is printed in each advertisement. The profit function for an advertis-

${ }^{6}$ In this paper, we implicitly assume that $D$ and $H$ are uncountable in the limit. 
ing message at $(R, k)$ is given by $\pi(R, k)$ as follows :

$$
\pi(R, k)=\theta(R, k)\left[R-R_{A}-k\right]-b,
$$

where $\theta(R, k)$ is the probability that a given advertising message at $(R, k)$ results in a contract, and thus $\gamma(R, k)=\theta(R, k) \mu(R, k)$. In the limit as $H$ approaches infinity, $\theta(R$, $k$ ) can be interpreted as the probability that an additional advertising message sent out at $(R, k)$ by any developer results in a contract. Likewise, in the limit as $H$ and $D$ get large, $\pi(R, k)$ can be interpreted as the change in profits received by a developer if he sends an additional message at $(R, k){ }^{7}$

Since $\theta(R, k) \leq 1$, developers would choose the combination $(R, k)$ which satisfies the following condition :

$$
R-R_{A}-k \geq b .
$$

Hence, we will focus on the triangle which is made by conditions (2.3), $R_{A}+b \leq R \leq Y$, and $0 \leq k \leq Y-R_{A}-b$. See fig. 2 for an illustration.

First of all, similar to Butters, we have the following two propositions.

Proposition 1. $\pi^{*}(R, k)=0$ for all combinations of $(R, k)$ that are advertised. Thus $\theta^{*}(R, k)=b /\left(R-R_{A}-k\right)$.

Proof. For large enough $D$ and $H, \pi(R, k)$ is a good approximation, say within $\varepsilon$, of the marginal product of advertising at $(R, k)$ [i.e., the change in profits given by an additional message at $(R, k)]$. If $\pi(R, k) \geq \varepsilon$, then some developer could improve his profits by sending an additional advertising message at $(R, k)$. If $\pi(R, k) \leq-\varepsilon$, then any developer

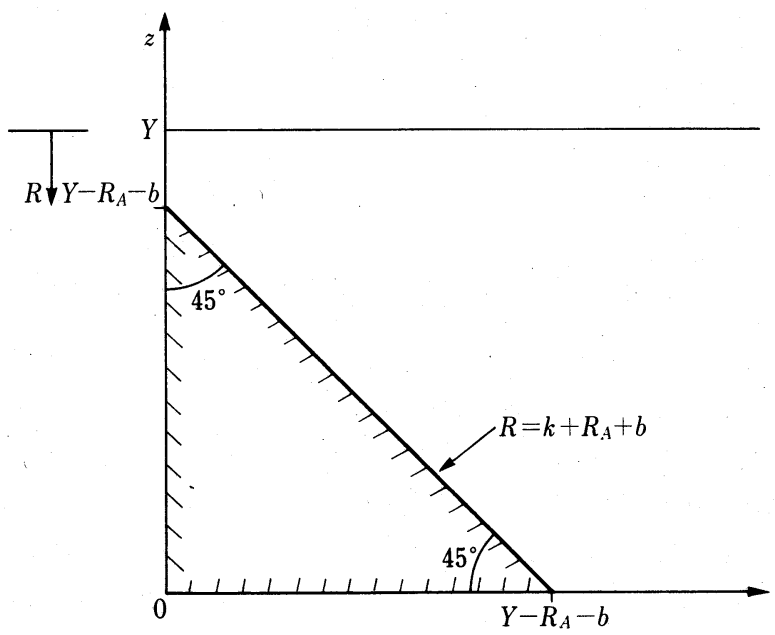

Fig. 2 The "choice space" of developers

7 In the limit, the probability that an extra advertising message of a developer will go to the household who has received the developer's other message, can be negligible. 
advertising at $(R, k)$ would do better to reduce that advertising message. Therefore, in equilibrium $|\pi(R, k)|<\varepsilon$ and in the limit $\pi^{*}(R, k)=0$.

(Q.E.D.)

Proposition 2. $\theta^{*}(R, k)=\exp \left[-M^{*}(R, k)\right]$.

Proof. As noted above, as $H$ increases, $\theta(R, k)$ may be approximated by the probability that an additional advertising message at $(R, k)$ would result in a contract. In other words, $\theta(R, k)$ is the probability that the message goes to a household having no other messages which are more favored than or indifferent to the message of $(R, k)$. Since we adopted the Poisson-type random process of allocating advertising messages, we have the equation

$$
\theta(R, k)=\exp [-M(R, k)] \text {. In the limit, } \theta^{*}(R, k)=\exp \left[-M^{*}(R, k)\right] .
$$

Proposition 3. In equilibrium, all combinations of $(R, k)$ that are advertised are on a curve which coincides with the "income expansion path (income-consumption curve)" of households.

Proof. Suppose, on the contrary, there are two advertising messages $a_{1}\left(R_{1}, k_{1}\right)$ and $a_{2}\left(R_{2}\right.$, $\left.k_{2}\right)$ such that $\left(R_{1}, k_{1}\right)$ and $\left(R_{2}, k_{2}\right)$ are on the same indifference curve $u=\tilde{u}$ and $a_{1} \neq a_{2}$, as depicted in fig. 3. Propositions 1 and 2 give,

$$
\begin{aligned}
& \frac{b}{R_{1}-R_{A}-k_{1}}=\exp \left[-M^{*}\left(R_{1}, k_{1}\right)\right] \text { for } a_{1}, \\
& \frac{b}{R_{2}-R_{A}-k_{2}}=\exp \left[-M^{*}\left(R_{2}, k_{2}\right)\right] \text { for } a_{2} .
\end{aligned}
$$

From the difinition of $M(R, k), M\left(R_{1}, k_{1}\right)=M\left(R_{2}, k_{2}\right)$ and thus $M^{*}\left(R_{1}, k_{1}\right)=M^{*}\left(R_{2}, k_{2}\right)$. Hence,

$$
\frac{b}{R_{1}-R_{A}-k_{1}}=\frac{b}{R_{2}-R_{A}-k_{2}} .
$$

Therefore,

$R-k=q_{1}$. for $\forall(R, k)$ which is advertised and such that $U(Y-R, k)=\tilde{u}$, where $q_{1}$ is a constant. This condition means that $a_{1}$ and $a_{2}$ must be on the same line $R$ $-k=q_{1}$. Next, suppose $a_{3}\left(R_{3}, k_{3}\right)$ is another advertising message such that $U\left(Y-R_{3}, k_{3}\right)$ $=\tilde{u}$, and $R_{3}-k_{3}=q_{2}>q_{1}$. From the definition, however, $M\left(R_{3}, k_{3}\right)=M\left(R_{1}, k_{1}\right)=M\left(R_{2}\right.$, $\left.k_{2}\right)$, and thus by Proposition $2, \theta^{*}\left(R_{3}, k_{3}\right)=\theta^{*}\left(R_{1}, k_{1}\right)=\theta^{*}\left(R_{2}, k_{2}\right)$. Since $R_{3}-R_{A}-k_{3}>R_{1}$ $-R_{A}-k_{1}=R_{2}-R_{A}-k_{2}$, the developer who sends $a_{1}$ or $a_{2}$ would be better off by changing his advertising policy from $a_{1} / a_{2}$ to $a_{3}$. This contradicts the assumption of equilibrium. Consequently, at equilibrium in the limit, $(R, k)$ in advertising messages is the solution of the following problem.

$(\tilde{R}, \tilde{k})=\underset{R, k}{\operatorname{argmax}}[R-k]$ subject to $U(Y-R, k)=\tilde{u}$. This is nothing other than the 


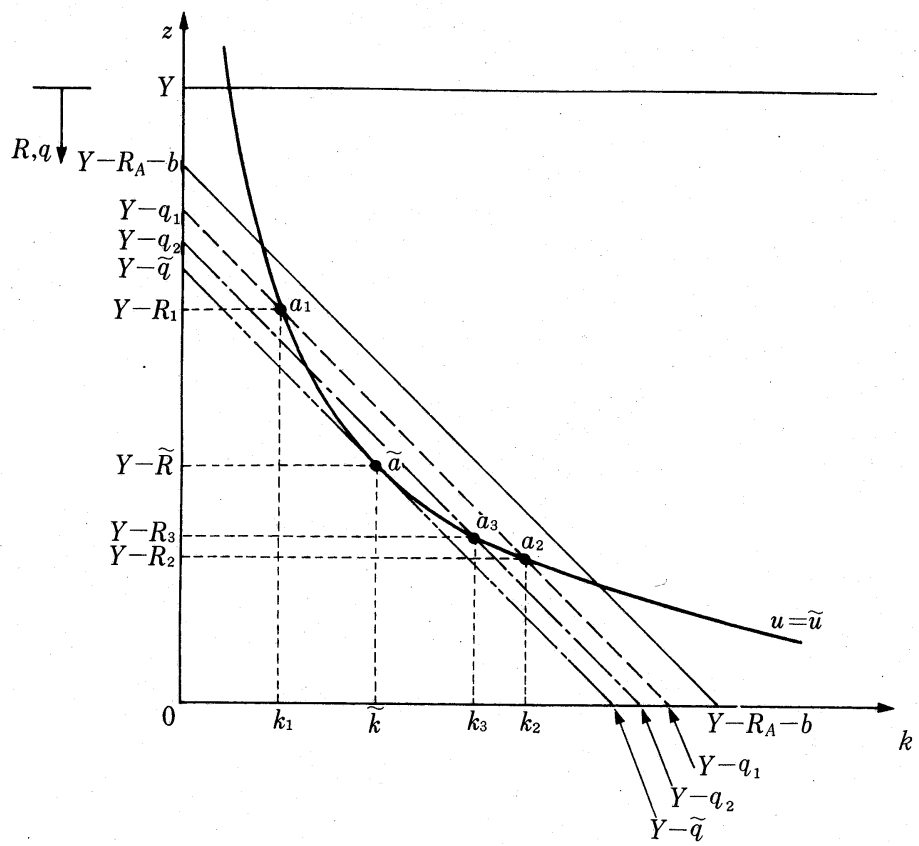

Fig. 3 The comparison among messages

tangency point of the indifference curve $u=\tilde{u}$ and the line $R-k=\tilde{q}$ where $\tilde{q}=\tilde{R}-\tilde{k}$ (i.e., $\tilde{a}$ in fig. 3). By changing $\tilde{u}$ appropriately, we can obtain a locus of $\tilde{a}(\tilde{R}, \tilde{k})$, which coincides with the (imaginary) income expansion path of households. ${ }^{8}$

(Q.E.D.)

Hence, $m(R, k)=\mu(R, k)$ and $c(R, k)=\gamma(R, k)$. Next, let us denote by $a_{0}\left(R_{0}, k_{0}\right)$ the tangency point of the indifference curve and the line $R=k+R_{A}+b . \quad 0$ denotes the origin [i. e., the point where $R=Y(z=0)$ and $k=0]$. Then we have the following proposition.

Proposition 4. In equilibrium, all combinations of $(R, k)$ on the "income expansion path" from $a_{0}$ to 0 are advertised.

Proof. (See fig. 4 for an illustration.)

Although the property of the "income expansion path" depends on the formula of the utility function, all combinations of $(R, k)$ satisfy the relation $R-k=q$. Thus we may restate this proposition as follows: all combinations of $(R, k)$ on the "income expansion path" which satisfy $\left[R-k=q\right.$, for $\left.q \varepsilon\left(R_{A}+b, Y\right)\right]$ are advertised in equilibrium.

8 In our model, we implicitly assume that households regard $k$ as the quality of residensial land and they do not conceive the price of infrastructure explicitly. Thus, in this paper, the "income expansion path" is only an imaginary concept. The path can be depicted if households know the price of infrastructure and choose the most favored combination $(z / R, k)$ under the budget constraint which is parametrically given. 


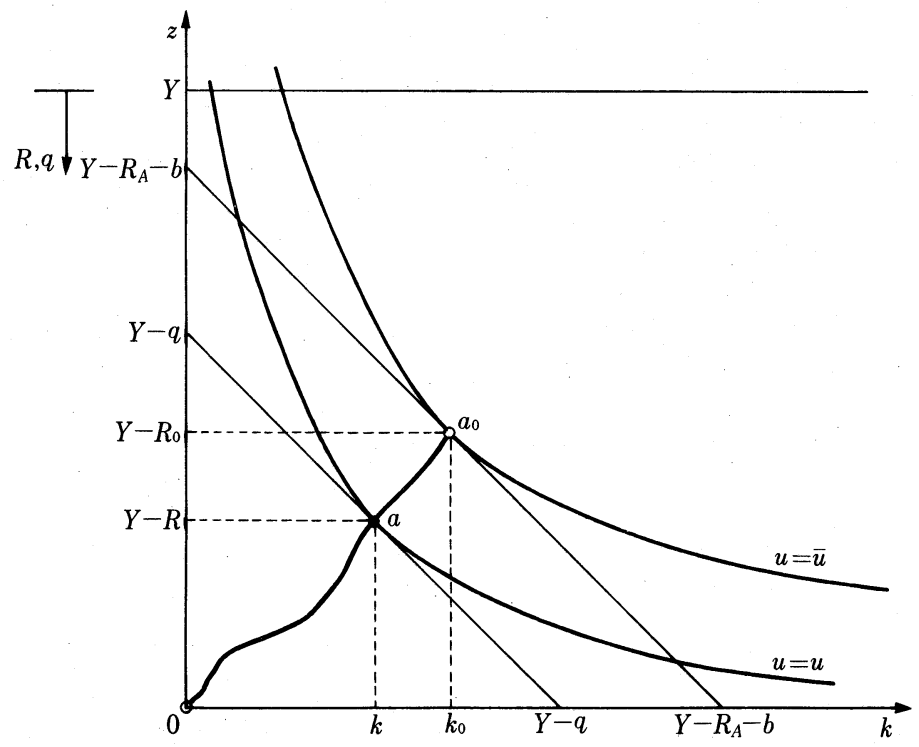

Fig. 4 The "income expansion path"

Then suppose, on the contrary, that all combinations of $(R, k)$ on the "income expansion path" which satisfy $\left[R-k=q\right.$, for $\left.q \varepsilon\left(q_{1}, q_{2}\right) \subset\left(R_{A}+b, Y\right)\right]$ are not advertised. Then a developer advertising $\left(R_{1}, k_{1}\right)$ such that $R_{1}-k_{1}=q_{1}$ could increase his profit per contract by $q_{2}-q_{1}$, by changing the message from $\left(R_{1}, k_{1}\right)$ to $\left(R_{2}, k_{2}\right)$ such that $R_{2}-k_{2}=$ $q_{2}$. This contradicts the assumption of equilibrium. An advertisement of $\left(R_{m}, k_{m}\right)$ such that $R_{m}-k_{m}=(\min q)$ generates a contract with certainty. Therefore $\theta\left(R_{m}, k_{m}\right)=1$, and applying Proposition 1,

$$
\left|\pi\left(R_{m}, k_{m}\right)\right|=\left|\left[R_{m}-R_{A}-k_{m}\right] \theta\left(R_{m}, k_{m}\right)-b\right|=\left|\left(R_{m}-k_{m}\right)-\left(R_{A}+b\right)\right|<\varepsilon
$$

for sufficiently large $H$ and $D$. Thus $\left(R_{m}-k_{m}\right)^{*}=R_{A}+b$, i.e., $(\min q)^{*}=R_{A}+b$. Hence $a_{0}$ is an end which corresponds to $(\min q)^{*}$, and thus $R_{m}^{*}=R_{0}, k_{m}^{*}=k_{0}$.

Corollary 1. If both of the composite good and the infrastructure are normal, $R \varepsilon\left(R_{0}, Y\right)$ and $k \varepsilon\left(0, k_{0}\right)$, for $\forall(R, k)$ which is advertised.

As noted above, it is convenient to use $q$ as a surrogate expression for $(R, k)$ where $q=R-k .^{9} \quad$ Thus, if we substitute $(q)$ into $(R, k)$ in all of our notations, we have the following proposition.

Proposition 5. The equilibrium joint distribution of rent and provision levels of infrastructure in advertising and contracts can be fully characterized by following equations and the formula for the utility function.

9 " $q$ " is a kind of projection of $(R, k)$ on the vertical/horizontal axis. Moreover, it can readily be shown that this projection is one-to-one. 


$$
\begin{aligned}
M^{*}(R, k): M^{*}(q) & = \begin{cases}0 & \text { for } q \leqq R_{A}+b \\
\log \frac{q-R_{A}}{b} & \text { for } R_{A}+b \leqq q \leqq Y\end{cases} \\
\mu^{*}(R, k): \mu^{*}(q) & = \begin{cases}0 & \text { for } q \leqq R_{A}+b \\
\frac{1}{q-R_{A}} & \text { for } R_{A}+b \leqq q \leqq Y\end{cases} \\
C^{*}(R, k): C^{*}(q) & = \begin{cases}0 & \text { for } q \leqq R_{A}+b \\
1-\frac{b}{q-R_{A}} & \text { for } R_{A}+b \leqq q \leqq Y\end{cases} \\
\gamma^{*}(R, k): \gamma^{*}(q) & = \begin{cases}0 & \text { for } q \leqq R_{A}+b \\
\frac{b}{\left(q-R_{A}\right)^{2}} & \text { for } R_{A}+b \leqq q \leqq Y\end{cases}
\end{aligned}
$$

Proof. From Propositions 1 and 2, we get $M^{*}(q)$. By differentiating $M^{*}(q)$ with respect to $q$, we have $\mu^{*}(q)$. By using $\theta^{*}(R, k): \theta^{*}(q)$ and $\mu^{*}(q)$, we get $\gamma^{*}(q)$. Then by integrating $\gamma^{*}(q)$, we have $C^{*}(q)$. The range is given in Proposition 4. Thus, if we know the formula for the utility function, by specifying the correspondence between $R$ and $k$, we may characterize the distributions in terms of $R$ and $k$.

(Q.E.D.)

\section{Discussion}

\subsection{Comparative Statics}

Surprisingly, as shown in Proposition 5, the equilibrium joint distribution of rent and provision levels of infrastructure (in advertising and contracts) in terms of $q$ are almost the same as the advertising and sales distribution of price obtained by Butters in terms of $p$ (price) (see Proposition 2.4. in Butters [2]). Hence, for the distributions in terms of $q$, we may fully utilize the results of the comparative static analysis by Butters.

\subsection{Welfare Analysis}

Let us examine the efficiency of the free land market under imperfect information. It should be noted that our concern is just with the number of total advertising messages in equilibrium.

The welfare standard may be defined as consumer (i.e., household) surplus plus developer profits, or gross benefits to households less production and advertising costs. Let us denote by $S_{H}$ the average surplus per household, and by $S_{D}$ the developer's profit per household. $\quad \bar{R}$ denotes the average contract rent and $\bar{k}$ the average contract provision level of the infrastructure. Define $\bar{q}=\bar{R}-\bar{k}$. As noted in Proposition 3, all combinations of $(R, k)$ which are contracted are on the "income expansion path." Hence, the surplus of an actual household who rents a lot of $(R, k)$ can be expressed as $Y-q$ where $q=R-k$. Therefore, $S_{H}$ is given as follows :

$$
S_{H}=[1-\exp (-M)][Y-\bar{q}],
$$

where $M$ is the number of advertising messages divided by the number of households, and 
thus $[1-\exp (-M)]$ is interpreted as the proportion of households who get the advertising messages. On the other hand, $S_{D}$ is given by

$$
S_{D}=[1-\exp (-M)]\left[\bar{R}-R_{A}-\bar{k}\right]-b M .
$$

Using the equilibrium condition $S_{D}=0$ and the difinition $\bar{q}=\bar{R}-\bar{k}$, the average welfare per household $W$ is obtained as

$$
W=S_{H}+S_{D}=S_{H}=[1-\exp (-M)]\left[Y-R_{A}\right]-b M .
$$

From the first-order condition $\partial W / \partial M=0$, we find the optimal number of advertising messages $M^{0}$ as follows : ${ }^{10}$

$$
M^{0}=\log \frac{Y-R_{A}}{b} .
$$

Clearly, $M^{0}$ coincides with $M^{*}(q=Y)$ which is the number of total advertising messages in equilibrium of the free market. Hence we can state the following proposition.

Proposition 6. The number of total advertising messages in equilibrium is socially optimal.

It is interesting to note that this result is the same as Butters'.

\section{Conclusion}

We have demonstrated that the provision level of infrastructure, as well as the land rent, may vary under imperfect information. ${ }^{11}$ Although these results are interesting, it is obvious that this paper is only an initial attempt in introducing economics of information into the analysis of land markets. Hence one may make criticisms from several points of view, indicating directions of further extension of our model.

First, this model is a kind of "one-shot game". In practice, however, households may store up information about land or reputations of developers. Although it would depend on the model adopted, we might conjecture that the range of distributions would be reduced and the number (and thus costs) of total advertising messages would be decreased by introducing the repeated and/or reputation game. Second, in practice, there are many sources of information about land. Especially, one may search for the land by spending some money and/or time. Although the analysis would be a complicated one, we may try to introduce other sources of information. Finally, even supplied by land developers, some kinds of residential infrastructure have the nature of local public goods. Thus, it is desirable to extend this model to the case in which local public goods are provided by land developers.

\footnotetext{
10 It can readily be shown that $\partial^{2} W / \partial M^{2}<0$.

11 As noted in footnote $2, k$ is the composite infrastructure. Hence, if the proportion of each good in the composite infrastructure varies according to the level of $k$, imperfectness of information also gives the variety of kinds of residential infrastructure.
} 


\section{References}

[1] Balcer, Y., "Equilibrium Distributions of Sales and Advertising Prices over Space," Journal of Economic Theory, Vol. 25, pp. 196-218, 1981.

[2] Butters, G.R., "Equilibrium Distributions of Scales and Advertising Prices," Review of Economic Studies, Vol. 44, pp. 465-491, 1977.

[3] Fujita, M., Urban Economic Theory, Chapter 5, Cambridge University Press, 1989.

[4] Grossman, G.M. and C. Shapiro, "Informative Advertising with Differentiated Products," Review of Economic Studies, Vol. 51, pp. 63-81, 1984.

[5] Nelson, P., “Advertising as Information," Journal of Political Economy, Vol. 82, pp. 729-754, 1974.

[6] Stathl, K., "A Note on the Microeconomics of Migration," Journal of Urban Economics, Vol. 14, pp. 318-326, 1983. 